\title{
Bone and hypopituitarism: not only a mass issue
}

\author{
Urszula T. Iwaniec
}

Published online: 29 June 2012

(C) Springer Science+Business Media, LLC 2012

Growth hormone (GH) is an important physiological regulator of bone growth and turnover [1]. Osteoblasts and chondrocytes express receptors for $\mathrm{GH}$, and $\mathrm{GH}$ has direct actions on differentiation and proliferation of these cells in primary cell culture. Congenital GH deficiency in humans and laboratory animals results in decreased bone growth and osteopenia. GH levels decrease with age, and this decline may contribute to common metabolic bone diseases, including postmenopausal and senile osteoporosis.

Hypophysectomy (hypox) in growing rats results in growth failure, hypogonadism, decreased white adipose tissue mass, and hypoleptinemia. Serum IGF-I levels plummet, as do mRNA levels for IGF-I in liver and bone. Longitudinal bone growth, periosteal bone apposition, and cancellous bone turnover are also drastically decreased. The volume fraction of cancellous bone falls, indicating that the magnitude of bone resorption exceeds formation. Concurrently, there is marked infiltration of bone marrow with fat. Hypox in rats also leads to low circulating levels of important gonadal, thyroid, adipocyte-derived, and adrenal hormones that influence energy metabolism and bone metabolism. GH replacement reinitiates weight gain, normalizes bone marrow adiposity, and restores near normal bone growth and turnover in the hypox animal. In contrast, administration of estradiol, IGF-I, thyroxine or cortisol to female hypox rats fails to reverse the effects of hypox on body weight gain and bone growth and turnover $[2,3]$. Taken together, these findings emphasize the

U. T. Iwaniec $(\bowtie)$

Skeletal Biology Laboratory, School of Biological and

Population Health Sciences, Oregon State University,

108 Milam Hall, Corvallis, OR 97331, USA

e-mail: urszula.iwaniec@oregonstate.edu importance of GH deficiency in mediating the skeletal abnormalities associated with pituitary ablation.

While the actions of pituitary hormones on bone growth and turnover have been extensively investigated, less is known regarding their actions on bone quality. In this issue of Endocrine, Bozzini et al. [4] report on the effects of pituitary ablation on biomechanical properties of cortical bone in rats. To circumvent differences in body size associated with GH deficiency, Bozzini et al. evaluated bone in rats of similar size but differing in age under the premise that animals of similar size would be exposed to similar load-induced strains allowing for determination of pituitary hormone deficiency on bone quality. Dramatic differences in extrinsic and intrinsic femoral biomechanical properties were observed between the control and hypox animals. Based on their results, the authors conclude: (1) that femurs from 1-year-old hypox rats (139 \pm 8 g body weight) are "overdesigned," being stronger and stiffer than femurs from the much younger control rats of similar body weight (138 $\pm 7 \mathrm{~g})$, and (2) that the observed differences are due to changes in the intrinsic properties of bone.

While there were major differences in the biomechanical properties of bone from the two groups, the analysis does not rule out an important contribution of age-related tissue level differences in bone quality. Bone is a composite material which is deposited as discrete layers by osteoblasts and as a consequence is neither homogenous in composition nor uniformly distributed. Bone is also porous containing osteocyte lacunae, a complex maize of canaliculi interconnecting osteocytes and cells lining bone, as well as vascular spaces emanating from both the periosteal and endocortical bone surfaces. The cortex of young female Sprague-Dawley rats weighing $\sim 150 \mathrm{~g}$ is more porous than that of 12-month-old skeletally mature rats (Fig. 1a, b) and contains calcified cartilage (Fig. 1c). 

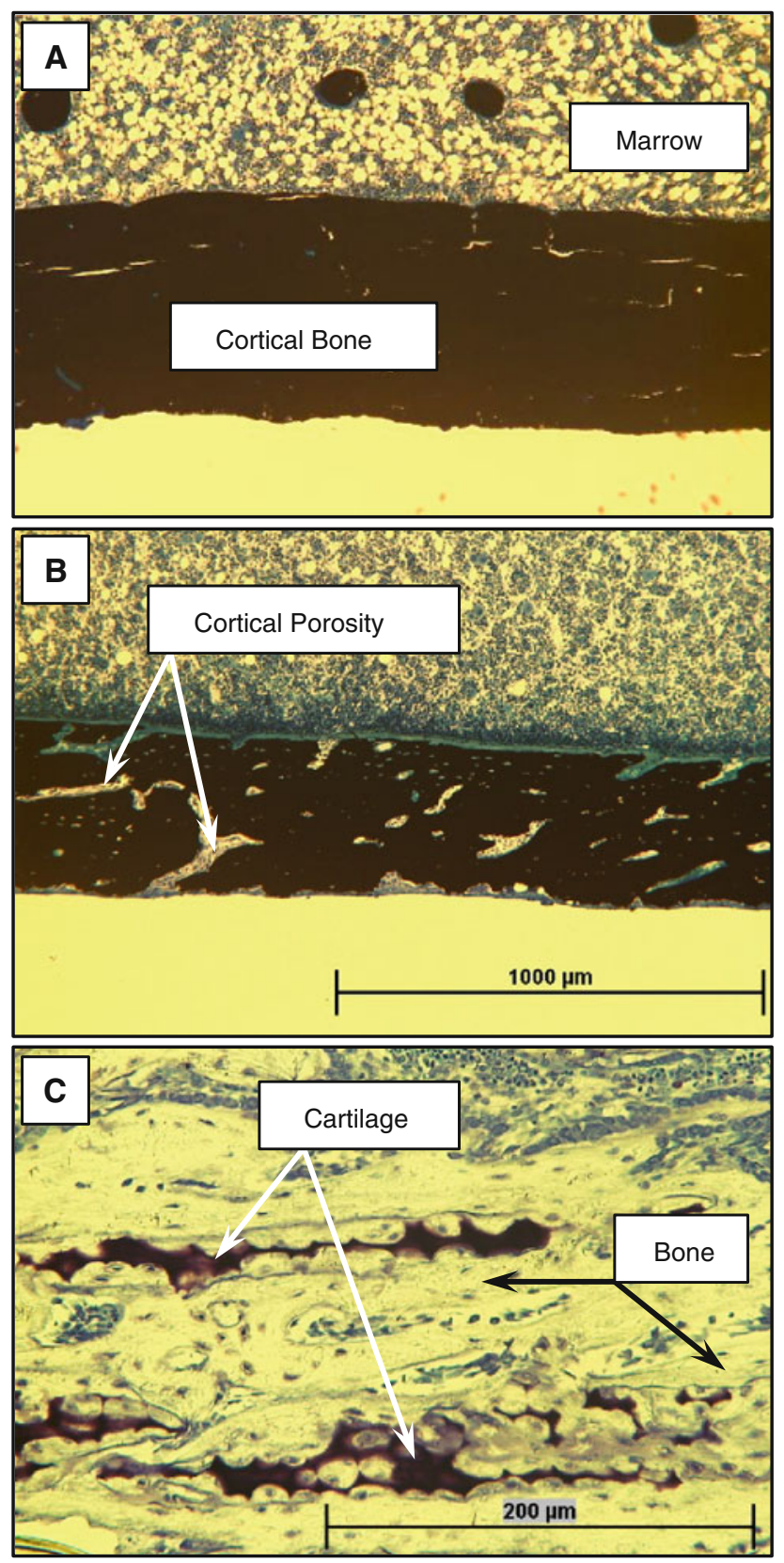

Fig. 1 Effect of growth on cortical bone. A comparison of cortical bone from a 1-year-old adult rat (a) and a $150 \mathrm{~g}$ growing rat (b). Please note the lower cortical thickness and greater cortical porosity in the younger animal. Please also note the extensive cartilage within the cortex of the young rat (c). Sections $(4-\mu \mathrm{m}$ thick) were stained according to the von Kossa method with a tetrachrome counterstain $(\mathbf{a}, \mathbf{b})$ or toluidine blue (c) [11]

Compared to bone matrix, calcified cartilage is less mineralized and less stiff. Thus, the increased mineral content, biomechanical strength, and stiffness of the aged hypox rats evaluated by Bozzini et al. may have been due, at least in part, to reduced porosity and replacement of calcified cartilage with bone matrix in the 12-month-old hypox animals.
Although similar weights of the animals in the two experimental groups would insure similar resting loads, skeletal adaptation is driven by dynamic (due to physical activity) rather than static loads [5] and there is no assurance that the ground reaction forces and load histories (magnitude and frequency of loads) experienced by hypox rats are comparable to those experienced by the much younger control rats. Furthermore, hormones impacted by hypox are important for skeletal response to weight bearing. Spaceflight and simulated spaceflight, for example, have been reported to result in skeletal resistance to the bone anabolic actions of GH [6]. Also, estrogen is reported to sensitize the skeleton to mechanical loading, and bone loss associated with menopause may be due to the reduced ability of the skeleton to respond appropriately to mechanical strain [7].

Bone mechanical properties are determined by the material properties of bone (largely independent of size), size, and distribution of mass (architecture). Because bone is anisotropic, mechanical and material properties of bone depend upon the direction and type of loading. Bozzini et al. used 3-point bending, a method in which bones generally fail at the point where the load is administered. Other common methods of inducing load failure include compression, torsion, and cantilever testing. Each method yields a unique value for bone mechanical and material parameters and the results may be discordant in terms of which treatment group exhibits superior quality. Hypox results in severe cancellous osteopenia in growing rats [8] which is likely to impact bone mechanical properties at any skeletal site containing cancellous bone. This is important because low trauma fractures typically occur at sites where cancellous bone microarchitecture is compromised. Furthermore, an increased prevalence of spinal deformities has been reported in adult patients with GH deficiency [9] which may be a consequence of altered material and biomechanical properties [10]. Thus, future studies should focus on evaluating biomechanical properties at additional skeletal sites using alternate testing methods.

\section{References}

1. A. Giustina, G. Mazziotti, E. Canalis, Growth hormone, insulinlike growth factors, and the skeleton. Endocr. Rev. 29, 535-559 (2008)

2. P.J. Menagh, R.T. Turner, D.B. Jump, C.P. Wong, M.B. Lowry, S. Yakar, C.J. Rosen, U.T. Iwaniec, Growth hormone regulates the balance between bone formation and bone marrow adiposity. J. Bone Miner. Res. 25, 757-768 (2010)

3. J.K. Yeh, M.M. Chen, J.F. Aloia, Effects of estrogen and growth hormone on skeleton in the ovariectomized rat with hypophysectomy. Am. J. Physiol. 273, E734-E742 (1997) 
4. C. Bozzini, E.O. Picasso, G.M. Champin, R.M. Alippi, C.E. Bozzini, Biomechanical properties of the mid-shaft femur in middle-aged hypophysectomized rats as assessed by bending test. Endocrine (2012). doi:10.1007/s12020-012-9616-0

5. C.H. Turner, Three rules for bone adaptation to mechanical stimuli. Bone 23, 399-407 (1998)

6. B.P. Halloran, D.D. Bikle, J. Harris, C.P. Autry, P.A. Currier, S. Tanner, P. Patterson-Buckendahl, E. Morey-Holton, Skeletal unloading induces selective resistance to the anabolic actions of growth hormone on bone. J. Bone Miner. Res. 10, 1168-1176 (1995)

7. R.T. Turner, Skeletal adaptation to external loads optimizes mechanical properties: fact or fiction. Curr. Opin. Orthop. 12, 384-388 (2001)
8. R.T. Turner, C.J. Rosen, U.T. Iwaniec, Effects of alcohol on skeletal response to growth hormone in hypophysectomized rats. Bone 46, 806-812 (2010)

9. D.B. Burr, Why bones bend but don't break. J. Musculoskelet. Neuronal Interact. 11, 270-285 (2011)

10. G. Mazziotti, A. Bianchi, S. Bonadonna, M. Nuzzo, V. Cimino, A. Fusco, L. De Marinis, A. Giustina, Increased prevalence of radiological spinal deformities in adult patients with GH deficiency: influence of GH replacement therapy. J. Bone Miner. Res. 21, 520-528 (2006)

11. U.T. Iwaniec, T.J. Wronski, R.T. Turner, Histological analysis of bone. Methods Mol. Biol. 447, 325-341 (2008) 